\title{
TECNOLOGÍAS AL ACECHO. MUTACIONES DEL VIAJE COTIDIANO EN UNA CIUDAD NO METROPOLITANA ${ }^{1}$
}

\author{
Lurking technologies. every day travel mutations in a non-metropolitan city
}

\author{
Tomás Errázuriz Infante* \\ Eduardo Valdés de la Fuente**
}

\begin{abstract}
RESUMEN
Como la gran mayoría de las ciudades chilenas, Talca ha aumentado significativamente el número de vehículos motorizados en circulación durante las últimas décadas. Este incremento ha sido acompañado por una gradual modernización de la infraestructura de tránsito. Si bien las cifras y diagnósticos generales sugerirían que Talca muestra -a menor escala- las mismas dinámicas que se observan en Santiago, este trabajo busca relevar procesos que son propios de un territorio urbano que no es metropolitano. Con este objeto, se recurre primero a un análisis de información estadística y documental que permite resaltar las particularidades de este proceso de motorización y modernización vial en comparación a Santiago. Segundo, se indaga en las experiencias cotidianas de viaje de 25 personas en Talca con el objeto de comprender prácticas distintivas asociadas a los viajes cotidianos y analizar de qué manera estas se ven afectadas por el nuevo entorno material.
\end{abstract}

Palabras clave: modernización, vehículos motorizados, movilidad, experiencia de viaje.

\footnotetext{
${ }^{1}$ Este trabajo fue posible gracias al apoyo de Conicyt a través del proyecto Fondecyt Regular $\mathrm{N}^{\circ}$ 1130695, titulado: "Transformación material y simbólica de las prácticas del transporte y movilidad cotidiana en una ciudad chilena intermedia".

* Académico, Universidad Andres Bello, Campus Creativo. Santiago, Chile. Correo electrónico: tomaserrazuriz@gmail.com

** Antropólogo, Universidad de Chile. Santiago, Chile. Correo electrónico: evaldesdelafuente@gmail.com Artículo recibido el 18 de diciembre de 2015. Aceptado el 11 de enero de 2017.
} 


\begin{abstract}
Like the vast majority of Chilean cities, Talca has significantly increased the number of motorized vehicles in circulation during the last decades. This increase has been accompanied by a gradual modernization of the transit infrastructure. Although the figures and general diagnoses would suggest that Talca shows - to a lesser extent- the same dynamics that are observed in Santiago, this work seeks to relieve processes that are typical of an urban territory that is not metropolitan. For this purpose, we first resort to an analysis of statistical and documentary information that allows highlighting the particularities of this process of motorization and road modernization compared to Santiago. Second, we investigate the daily travel experiences of 25 people in Talca in order to understand the distinctive practices associated with everyday trips and to analyze how they are affected by the new material environment.
\end{abstract}

Keywords: modernization, motor vehicles, mobility, travel experience.

\title{
INTRODUCCIÓN
}

La recurrencia de un viaje cotidiano por la ciudad y el avance sostenido que presentan los vehículos a motor como principal medio de desplazamiento es un fenómeno contemporáneo que ha permitido naturalizar una serie de dispositivos técnicos como parte del paisaje urbano. Además de los vehículos mismos (automóviles, autobuses, motocicletas, etc.) la tendencia ha sido hacia una proliferación de otros artefactos como semáforos, señalética, sistemas automatizados de control del tránsito, vías especializadas, etc., a cada uno en su momento se le ha vestido de las promesas del progreso urbano, que anuncian mayor eficiencia, velocidad, seguridad y control. Ideados y legitimados desde aquellas ciudades que lideran los procesos de modernización, estos artefactos -casi sin alteracionesestablecen complejas redes (Urry, 2004) y avanzan indistintamente en conquista de nuevos territorios, repitiendo un movimiento constante y civilizatorio desde los centros de poder hacia las periferias globales.

Pero ¿qué sucede cuando estos objetos llegan a las ciudades más alejadas, a aquellos poblados en donde la vida guarda escasa relación con aquella que anima a la gran metrópolis? ¿Qué sucede cuando las promesas civilizatorias y los ideales de orden pensados desde áreas metropolitanas se instalan, por ejemplo, en la ciudad media o pequeńa de base agrícola? ¿Qué alcance tienen estos nuevos dispositivos sobre las prácticas cotidianas de movilidad o sobre las formas tradicionales de sociabilidad?

Responder a estas interrogantes implica necesariamente posicionarse críticamente frente a la larga tradición de los estudios urbanos y su natural 
predilección por la metrópolis. Aunque en sus orígenes y durante el transcurso del siglo veinte esta atención puede justificarse debido a que fueron esas ciudades las que experimentaban las más visibles y novedosas transformaciones, actualmente las ciudades que no cumplen con esta denominación experimentan cambios igual o más profundos que las metrópolis. Se suma el hecho de que la mitad de la población urbana mundial vive en localidades que tienen menos de $500 \mathrm{mil}$ habitantes (United Nations, 2015). Además, según el Observatorio de Ciudades Mundiales de ONU-Habitat, para el caso de América Latina, serían estas mismas ciudades las que más rápido están creciendo (United Nations, 2014).

Frente a estas circunstancias resulta preocupante que la investigación urbana siga secuestrada por la gran ciudad y que otras realidades urbanas se observen habitualmente a través de la lupa metropolitana. Esta lupa tiende a reducir cualquier diferencia a un problema de escala, omitiendo la situación geopolítica y desconociendo la historia y cultura de los territorios. Así, cuando la variable es solo el tamańo, la lupa permite leer e interpretar los fenómenos de ciudades no metropolitanas desde los conocimientos adquiridos en base a la metrópolis. Aunque, es probable que existan muchos fenómenos urbanos que pueden entenderse desde claves comunes, la escasez de investigación en el área de lo "urbano, no metropolitano" favorece una proliferación de los espejismos y fórmulas únicas.

En el campo específico del transporte urbano, históricamente, la ciudad de Santiago, ha concentrado la atención de las autoridades y especialistas (Errázuriz, 2010). Resulta preocupante, en este sentido, buscar literatura académica sobre las transformaciones del transporte y la movilidad durante las últimas décadas que no tengan por caso de estudio la ciudad de Santiago, y descubrir que la producción es mínima o inexistente.

Esta falta de atención contrasta con la intensidad de los cambios en el transporte y la movilidad en regiones, en muchas ocasiones incluso mayores que en la capital. A las variaciones en el parque vehicular, se agregan otras problemáticas que históricamente han sido asociadas a los grandes centros urbanos, pero que hoy día afectan sin distinción a ciudades de tamaño intermedio, tales como la contaminación ambiental o la congestión vehicular.

En las líneas que siguen se examinará el proceso de penetración de los artefactos y dispositivos del tránsito moderno en una ciudad no metropolitana durante las últimas décadas. Se busca comprender qué particularidades distinguen este proceso específicamente en Talca y esbozar en qué medida este se aleja del recorrido experimentado en Santiago. Para alcanzar este objeto se proponen dos lecturas complementarias que permiten aproximarse al problema desde distintas perspectivas. La primera de estas se refiere específicamente al reconocimiento y caracterización de los nuevos objetos y tecnologías de la movilidad que arriban 
a este territorio en particular. La segunda pone en relación a los nuevos objetos tecnológicos con un conjunto de prácticas cotidianas, identificando y analizando eventuales influencias entre unas y otras.

Para llevar a cabo este análisis, se ha recurrido a un conjunto de fuentes, entre los que se incluye material estadístico de los registros del Instituto Nacional de Estadística, de la Asociación Nacional Automotriz de Chile, la Encuesta Origen Destino y una Encuesta de Movilidad cotidiana aplicada a 800 personas residentes de la ciudad de Talca; material histórico documental extraído de la revisión de los principales medios de prensa local, así como de otros documentos varios; y entrevistas en profundidad que se realizaron a 25 personas residentes en Talca y sus alrededores.

\section{LAS TECNOLOGÍAS / ¿MODERNIZACIÓN DE SEGUNDA?}

A través de los principales medios de prensa escrita y televisiva nos hemos acostumbrado a escuchar que el parque de vehículos motorizados en Santiago no ha cesado de crecer a un ritmo frenético durante las últimas décadas. No obstante, al revisar cifras desde mediados de la década del setenta en adelante, el principal crecimiento se ha producido lejos de la Región Metropolitana (RM). Mientras entre 1975 y 2012 la RM aumentó su parque vehicular motorizado en 7,2 veces, el resto del país lo hizo en 9 veces. En el caso específico de la provincia de Talca, el aumento fue de más de 11 veces. Ahora bien, si solo se consideran automóviles y motocicletas, la relación entre la Provincia de Talca y la RM se incrementa aún más llegando a 15,1 y 7,8 veces, respectivamente; es decir, casi el doble (INE, 19752012).

Cuando se observa específicamente los cambios en el parque de vehículos motorizados durante el último quinquenio, se refuerza esta tendencia. Mientras la Región Metropolitana aumentó entre el 2009 y 2014 en un 39,9\%, las restantes regiones del país lo hicieron en un 49,8\%. La diferencia se acrecienta si excluimos de esta última cifra a las otras áreas metropolitanas (La Serena, Valparaíso y Concepción), alcanzando entonces un 52,2\%. En base a estos datos podemos afirmar que la mayor parte del crecimiento del parque de vehículos a motor se ha producido no solo fuera de la Región Metropolitana, sino además alejado de los principales núcleos urbanos. Sin embargo, un análisis detenido confirma la necesidad de estudiar caso a caso. Específicamente en la Provincia de Talca, las dos comunas que muestran los mayores índices de crecimiento del parque de vehículos motorizados en los últimos cinco años, Maule y San Clemente con un $113,3 \%$ y $87,7 \%$ respectivamente, son las dos comunas hacia las cuales Talca se ha expandido en forma más clara en este mismo período (INE, 2009; INE, 2014). 
Esta tendencia de mayor intensidad de los cambios en áreas no metropolitanas, está en concordancia con la intensidad del crecimiento demográfico en las ciudades de Chile. No son Santiago, Concepción o Valparaíso los centros urbanos que más han crecido sino que son las ciudades intermedias con índices anuales que en los últimos 5 ańos triplican los que se presentan en las áreas metropolitanas (United Nations, 2015).

De tal modo, no solo es una cuestión de cantidad. Hoy más que nunca, se sabe que el tiempo de uso es un factor crítico en el desempeńo funcional y simbólico de los objetos tecnológicos. Cuando se observa la variable de antigüedad del parque vehicular, la Asociación Nacional Automotriz de Chile confirma que durante el 2013 la Región Metropolitana concentró el 58\% de las ventas de vehículos nuevos, pese a que el parque de vehículos en circulación en este lugar solo representaba un $41 \%$ del total nacional. En otras palabras, los datos sugieren que un porcentaje importante de los vehículos que llegan a otras regiones son usados. Efectivamente, cuando se analiza el parque total de vehículos livianos y medianos en la Región Metropolitana, estos alcanzan un promedio de 7,8 años de antigüedad, mientras que en la Región del Maule este promedio asciende a 11,6 años y en el país a 9,5. (ANAC, 2014).

Solo considerando la antigüedad de los vehículos, se comprende que la Región del Maule, con el promedio de sueldos más bajo del país (Superintendencia de Pensiones, 2015), sea la misma que en la última década contempla junto con la región de Coquimbo, la mayor alza promedio del parque automotriz al año, que bordea el 12\%. La tendencia en Chile demuestra que el automóvil privado ha dejado de ser un bien exclusivo y su uso se extiende en forma progresiva hacia los segmentos más bajos (Ureta, 2009). Se podría afirmar que el nivel socioeconómico ya no se mide necesariamente por el acceso al bien, sino por el número de vehículos por hogar y la marca y año del automóvil al cual se accede. A su vez, los parámetros que la gente utiliza para determinar el valor de un automóvil se han diversificado, especialmente cuando se trata de autos usados. El amplio mercado de dispositivos, piezas y procedimientos que permiten personalizar la apariencia y el funcionamiento de un automóvil al gusto de su propietario, favorece la generación de nuevos significados asociados al artefacto tecnológico que trascienden a los tradicionales que confieren la marca, el modelo y el año de fabricación.

Si en el parque de vehículos motorizados existe una diferencia considerable en la antigüedad entre la Región Metropolitana y la Región del Maule, la brecha se incrementa cuando solo se observa a los autobuses. La antigüedad promedio de los buses del transporte público en la Región del Maule es la más alta del país llegando a 13,4 años, mientras la RM mantiene la más baja con 7 años (ANAC, 2014).

En consecuencia, no solo llegan a las provincias mayor cantidad de vehículos y en peores condiciones, sino que además se instalan en un contexto 
que la mayoría de las veces no está acondicionado para recibirlos. La preeminencia del centro como principal destino de la gran mayoría de los viajes, la persistencia de la antigua estructura de calzadas y veredas angostas y de usos tradicionales no siempre asociados a la movilidad, son elementos que suelen hacer crisis ante un aumento significativo de los viajes privados motorizados. Esta crisis se manifiesta principalmente en problemas de conectividad entre el centro y los alrededores, en la proliferación de estacionamientos informales en las áreas centrales, en situaciones de congestión vehicular, aumento de tiempos de viaje, mayor número de accidentes, etc. Estas condiciones no solo distinguen el entorno cotidiano de los viajes en Talca, sino que pueden observarse con escasas variaciones en otras ciudades de escala parecida en Chile (CEHU, 2011; Eltit, 2011). El resultado es que ciudades pequeñas, en donde los viajes no debieran tener mayores complicaciones, comienzan a padecer aquellos problemas que históricamente han sido asociados a las grandes ciudades. Una editorial del principal diario de Talca señalaba el 2004 que resultaba sorprendente que "una urbe de sólo 200 mil habitantes sea víctima de asfixiantes congestiones vehiculares que perfectamente están a la altura de ciudades más grandes y de crecimiento explosivo" (El Centro, 02 de septiembre de 2014).

Como respuesta, autoridades y expertos no dudan en proyectar e implementar las soluciones que a estos problemas se les ha dado en la capital. Es el caso de mejoras en las tecnologías de semaforización, la implementación del nuevo Sistema Operativo de Control de Tránsito, o de diversas obras de infraestructura vial que prometen terminar con la falta de conectividad ocasionados por la carretera, la línea del ferrocarril o algún curso fluvial que el desregulado crecimiento urbano transformó repentinamente en barrera. Por diversos motivos los anhelos modernizantes, que tienen por modelo a la ciudad capital, con dificultad trascienden su discurso. Cuando esto ocurre, su materialización es por lo general acotada, simbólica, puntual y no conduce a una transformación integrada.

No obstante, cuando se observa en retrospectiva las últimas décadas, es evidente el vuelco que ha experimentado el entorno construido hacia el paradigma de una movilidad motorizada. Si bien la cantidad de vehículos-automóviles, buses y otros a motor- siguen siendo el espectro más visible de esta transformación, también la proliferación de señalética, semáforos, paraderos, pero sobre todo la pavimentación de calles y caminos interurbanos, así como las obras de caminos de acceso y circunvalación, son distintivos de un nuevo paisaje de la movilidad en Talca.

De tal modo, este nuevo entorno material tendrá resultados muy distintos dependiendo de si se encuentra dentro o fuera de la ciudad. El incesante movimiento del terminal de buses de Talca es el mejor testimonio de cómo la pavimentación y construcción de caminos interurbanos, así como el aumento sostenido en la oferta de transporte público interurbano han transformado dramáticamente 
las posibilidades de movilidad cotidiana de los poblados vecinos. Una mirada a los destinos que se pueden alcanzar desde este terminal y especialmente a las frecuencias con que salen actualmente los buses a cada localidad es indicativo del tráfico constante que existe entre Talca y otras localidades de la región. A San Clemente $(20 \mathrm{~km})$ salen buses cada 5 minutos, a Pelarco $(26 \mathrm{~km})$ cada 8 minutos. Si se quiere ir a Linares $(53 \mathrm{~km})$, San Rafael $(18 \mathrm{~km})$, Duao $(20 \mathrm{~km})$, Curicó (67 $\mathrm{km})$ Maule $(14 \mathrm{~km})$ existe movilización colectiva cada 10 minutos, mientras a Constitución $(111 \mathrm{~km})$, Curepto $(75 \mathrm{~km})$ y Cauquenes $(104 \mathrm{~km})$, la frecuencia es siempre de menos de una hora. Finalmente a Santiago $(256 \mathrm{~km})$ salen un promedio de 5 buses por hora.

Este intenso flujo entre Talca y sus alrededores está en directa relación con la transformación de la infraestructura vial. Así, observamos que mientras en 1981 solo el 13\% de la red vial de la Región del Maule se encontraba pavimentada, para el 2013 este porcentaje había aumentado al 34\%. Durante este mismo período, los caminos de ripio se mantuvieron en la mitad del total, mientras que los de tierra disminuyeron de un 38\% a un 15\%. Pero no solo se pavimentan vías preexistentes, sino que también aumentó la extensión de la red vial. Este aumento se concentra casi exclusivamente en el período 1981-1999, pasando de 4.226 a 7.720 kilómetros (Riveros, 2013).

La experiencia es distinta en la ciudad. Las promesas de conectividad, velocidad, acceso, eficiencia, que una carretera interurbana y una flota de buses pueden cumplir, difícilmente se concretan dentro de la ciudad con intervenciones similares. El acceso a un vehículo motorizado no es necesariamente sinónimo de mayor velocidad y menores tiempos de viaje; el ensanche de una calle no garantiza el fin de la congestión; la colocación de semáforos y señalética, no necesariamente incrementa la fluidez del tráfico. De manera similar sucede con los medios de transporte público que si bien han aumentado en su cobertura y cantidad, este mayor número no necesariamente se traduce en eficiencia y conectividad. Un análisis detallado de los distintas líneas de colectivos y autobuses urbanos en Talca muestra que lo habitual son recorridos intrincados que no parecieran tener entre sus objetivos buscar los trayectos de menor distancia entre dos puntos.

En una serie de 25 entrevistas realizadas a personas que viven y viajan cotidianamente en la ciudad de Talca ${ }^{2}$, todos los entrevistados al ser consultados por cómo habían cambiado sus viajes en las últimas décadas reconocían de inmediato el rápido desarrollo que habían experimentado las formas de locomoción motorizada y el entorno material. Sin embargo, la gran mayoría de los testimonios coincidía en

\footnotetext{
${ }^{2}$ Las entrevistas fueron realizadas dentro del contexto de la tesis para obtener el grado de Antropólogo en la Universidad de Chile, titulada: "Movilidad cotidiana y representaciones sociales de la ciudad fuera del contexto metropolitano. El caso de la ciudad de Talca" de Eduardo Valdés de la Fuente.
} 
que esta modernización no se había traducido necesariamente en un mejor viaje, o en uno más eficiente. Uno de los argumentos que más se repiten fue que si bien había aumentado la oferta de medios de transporte, los recorridos se volvían más lentos y más peligrosos y el viaje era cada vez motivo de mayor estrés.

\section{LAS PRÁCTICAS / ¿LA CIUDAD QUE DUERME?}

Talca, a los ojos de los metropolitanos, se podría decir que duerme, se mueve a paso lento, lejos del frenesí y el nerviosismo de la gran urbe. No solo se estima la siesta, el descanso y la tranquilidad, sino que se cultivan una serie de valores tradicionales que por el momento parecieran resistir a la vorágine del capitalismo. Existe un sensación de que los tiempos cotidianos y las relaciones interpersonales, no estarían aún instrumentalizadas en función del dinero como tempranamente advierte Simmel (2005) para la metrópolis y, en cambio, persistirían costumbres e ideales que desde una perspectiva económica clásica, atentarían contra la eficiencia, la productividad y el crecimiento. En palabras de Greene (2014), son lugares donde "el capitalismo tiene horario", donde aún prima una manera de producir, vender y consumir, que en muchos sentidos no ha variado por décadas.

A este Talca llegan en gran número los artefactos motorizados de segunda mano y sus instrucciones para construir la modernidad. El objeto mecánico se inserta en un contexto socio-cultural que no necesariamente sigue las pautas y lógicas de producción y acumulación. Ejemplo de esto es que en Talca el 85\% de sus habitantes habitualmente almuerza en sus casas (CEUT, 2014), habiendo por tanto un porcentaje significativo que realiza cuatro viajes diarios en vez de dos.

El ideal de la movilidad urbana como flujo eficiente, característico de ciudades metropolitanas, pareciera no aplicar en una ciudad como Talca, donde las interrupciones y pausas ocupan un lugar central en el ritmo del quehacer cotidiano. Detener el automóvil a media cuadra para esperar a alguien o bajarse a comprar algo, sin mayor preocupación por las consecuencias en el flujo vial, es una práctica habitual que desconcierta a quienes llegan desde la capital. La interrupción como práctica naturalizada es posible, por cierto, en una ciudad en la cual el $80 \%$ de sus habitantes demora menos de 20 minutos en su viaje al trabajo ("Encuesta de Movilidad", 2014).

Laconversación, queaojos de Humberto Gianinni (2004) implicael principal acto de interrupción y quiebre de la rutina de movilidad cotidiana, adquiere un lugar central en los espacios de tránsito en Talca. El 68\% de quienes viven en Talca afirman que durante sus trayectos cotidianos siempre o habitualmente conversan con personas que conocen y un 30\% lo hace con desconocidos. La hipertrofia del sentido de la vista a la que refieren Simmel y Wirth como rasgo distintivo de las 
relaciones sociales en la metrópolis, se diferenciaría de las ciudades más pequeñas donde la capacidad de escuchar al otro cumple un papel fundamental (Joseph, 2002). En esta misma encuesta se les consultó a quienes viajan en la locomoción colectiva la cantidad de personas conocidas con las que se encuentra en uno de sus trayectos habituales. Sorprende que un $47 \%$ se encuentra con más de tres personas y un $21 \%$ con más de nueve. Solo un $9 \%$ afirmó que habitualmente no se encuentra con nadie conocido en su trayecto. ("Encuesta de Movilidad", 2014). Con respecto a la recurrencia de la conversación en estos encuentros, el $47 \%$ de los encuestados sostuvo que habitualmente habla con personas que conoce durante su trayecto cotidiano, mientras un $51 \%$ habla habitualmente con desconocidos y un $56 \%$ afirma que sin querer le toca escuchar conversaciones ajenas. Esta disposición a la interacción social queda además reflejada en la atención visual de quien viaja sobre su entorno inmediato. Si bien no contamos con referentes para otro tipo de ciudades, es indicativo de la valoración de la privacidad en el espacio público del viaje, el hecho de que un $46 \%$ de los encuestados sostenga que habitualmente se siente observado por otros pasajeros y un 50\% reconozca sin problemas que habitualmente se dedica a observar a otras personas mientras viaja ("Encuesta de Movilidad", 2014).

Si una de las características de la megalópolis es que proporciona experiencias de desconocimiento que incentivan la construcción de imaginarios y prejuicios que tienen por base interacciones sociales evasivas y fugaces (García Canclini, 2010), se podría postular que la ciudad pequeńa, por su escala y la mayor disposición a la sociabilidad cara a cara, favorecería las experiencias de conocimiento e interacción 'real'. A diferencia de lo que puede generar el viaje en Metro o en el Transantiago, en Talca, la micro o el colectivo, son en gran medida espacios de encuentro con lo conocido, lo predecible, desde donde es factible apreciar y recorrer lugares que resultan generalmente familiares.

Consultados sobre sus prácticas cotidianas de movilidad, un grupo de 25 entrevistados que habitualmente transitan por Talca, confirmaron la importancia que tiene el encuentro y la conversación presencial (cara a cara) en sus recorridos por la ciudad. No obstante, no se trata de una costumbre transversal a todas las formas de transporte. Esta práctica es más frecuente entre quienes usan de preferencia el transporte público y la caminata como medios principales, y en cambio es menos frecuente entre ciclistas y prácticamente inexistente entre quienes viajan en automóvil.

Lejano de la noción de anonimato que tradicionalmente ha sido asociada a la gran ciudad desde los estudios urbanos, en Talca es habitual el reconocimiento e intercambio social en el viaje. Una de las entrevistadas señala en este sentido: "me entretengo, si me encuentro con una amiga me pongo a conversar nos vamos caminando". No es extraño para quien se moviliza a diario en Talca, que al subir 
a una micro la persona salude a alguno de los pasajeros y eventualmente inicie una conversación. Tal como comenta otro de los entrevistados, "la mayoría de las veces te encuentras con personas conocidas, entonces te vas conversando todo el viaje con ellos". Esta noción del viaje como un espacio abierto a la interacción con desconocidos pareciera ser especialmente frecuente entre mujeres adultas, quienes ven el tiempo de viaje como oportunidad de distracción y entretenimiento.

Ya, como es viajar... de repente es buena onda, nos ponemos a conversar y todo, pero de repente vamos todos callados y sentados. O gente que toca al lado también, a veces se conversa, a veces no.

Pero la interacción no se limita a otros pasajeros, sino que también son habituales los intercambios entre choferes y pasajeros. Trascendiendo la cordialidad habitual del saludo, el agradecimiento o la despedida, los entrevistados reconocen entablar en forma recurrente conversaciones más extendidas, en donde se comenta sobre temas generales como el clima, el trabajo o se pregunta por posibles personas que puedan existir en común. También es habitual aquellos que sostienen que ni siquiera necesitan indicarle a los choferes donde se bajarán, ya que tal como afirma una de las entrevistadas "yo utilizo tanto las líneas que ya me conocen". En esta relación personalizada, favorece un clima de confianza y seguridad en los viajes cotidianos, puesto que la conversación disminuye la incertidumbre y el riesgo de lo desconocido y favorece una mayor apropiación de estos espacios.

El grupo con menos inclinación a iniciar algún tipo de interacción con desconocidos son los entrevistados más jóvenes, quienes prefieren realizar otras actividades durante el viaje (escuchar música, estudiar, chatear). Esta actitud de viaje ha sido reconocida por Jirón (2015) como una modalidad capullo que implicaría estrategias conscientes de aislamiento y protección de un espacio de intimidad. Dentro de este grupo, la mayoría declara que solo conversa con conocidos, mientras aquel a quien no se conoce permanece como un extraño:

Si yo veo dos personas hablando en una micro, yo asumo que se conocen... me sería muy extraño el encontrar a dos personas desconocidas hablando, es más, si alguien desconocido me habla lo encontraría súper extraño.

A diferencia de quienes usan de preferencia el transporte público y la caminata como medios principales, entre quienes regularmente se mueven en bicicleta, las respuestas fueron más variadas. Mientras algunos declararon que evitaban los encuentros, otros señalaban que si bien saludaban a otros en su camino, raramente se detenían a conversar. En este sentido, la necesidad de concentración durante el manejo en bicicleta es un factor de creciente importancia en calles que 
han aumentado el flujo, la velocidad e intensidad de vehículos motorizados. Uno de los entrevistados que anda regularmente en bicicleta, ante la pregunta sobre encuentros durante el viaje responde negativamente, "a no ser que me griten, me llamen y ahí tengo que parar”. Este testimonio es indicativo de la condición abierta de la bicicleta hacia el entorno, muy distinta a las posibilidades de aislamiento que ofrece el automóvil. Por último también existían casos de ciclistas cuyos testimonios confirmaban que sus posibilidades de sociabilidad no se veían reducidas, sino incrementadas por la bicicleta.

Cuando hay alguna persona que quiera hablarte te paras sin problemas, no hay que buscar estacionamiento, entonces tiene hartas ventajas.

Distinto es el caso de quienes se trasladan en automóvil en donde existe coincidencia en una cierta desconexión con el entorno social del viaje. Consultados por posibles encuentros, la negativa es lo más habitual, aludiendo a justificaciones como la necesidad de concentrarse, de manejar bien, o que simplemente el interés por otros asuntos como escuchar la radio. Una entrevistada incluso alude que varias veces le pasa que le dicen "oye andabai en auto y no me saludaste". A diferencia de la bicicleta, el automóvil, a través de su diseño hermético y diversos dispositivos de control del ambiente interior, brinda la posibilidad de reducir y mediar el vínculo con el entorno recorrido. Más aún, varios de ellos, al ser consultados, seńalaban a que cuando ocurrían eventuales interacciones, se trataba más bien de desavenencias y situaciones de conflicto con otros conductores o peatones.

Las posibilidades restringidas de interacción social que favorece el automóvil, se vuelven un factor relevante en la medida en que este modo de desplazamiento adquiere protagonismo en la ciudad. El fuerte incremento en el número de automóviles entre los residentes de Talca y los habitantes de las localidades cercanas durante los últimos cinco años; la proliferación de nuevos barrios en la periferia y el despoblamiento del centro acelerado por los daños del terremoto; la consolidación de un comercio mayorista que ofrece una gran variedad de productos en lugares a los que se accede principalmente en automóvil; la priorización del uso de dineros públicos para nuevos caminos, ensanches y pavimentaciones, en detrimento de mejoras en el sistema de transporte público, fomento del uso de la bicicleta, o mejoras en las veredas; son todos factores que por una parte incentivan el uso de más automóviles, y por otra -directa o indirectamente-, empeoran las condiciones de circulación, aumentando tiempos de viaje, niveles de estrés y frustración asociados a los viajes en general.

La centralidad que adquiere el automóvil, que a simple vista pareciera manifestarse solo en mayor cantidad de tacos, tiempos más largos de viaje y quizás en problemas de contaminación, comienza a transformar lentamente y a veces de 
manera casi imperceptible ciertas prácticas cotidianas distintivas de la vida urbana no metropolitana. La siesta, la importancia del almuerzo familiar, el saludo, la conversación de paso, son costumbres cuya persistencia depende de rutinas y de un cierto entorno construido y social que las posibilita.

Aunque la práctica de volver a almorzar en casa es todavía extendida entre quienes viven en Talca, los tiempos cada vez más largos de desplazamientos generan un desincentivo. Un claro ejemplo de esta situación se ve entre funcionarios de la Universidad Católica del Maule, localizada a $5 \mathrm{~km}$ al oriente de la Plaza de Armas. El viaje al centro a la hora de almuerzo que antes demoraba 10 minutos en automóvil, actualmente toma alrededor de 20 . Si se toma esta distancia como referente promedio, implicaría que si el trabajador tiene 1 hora de colación, al menos 40 minutos se destinarán al viaje. La aparición reciente de al menos seis locales que ofrecen almuerzos a los trabajadores de este establecimiento y la alta demanda que tienen por parte de los funcionarios es un claro testimonio de esta transformación.

El aumento de los tiempos de viaje, la recurrencia de los problemas de congestión vehicular y la incertidumbre respecto a las condiciones del trayecto, también generan nuevas prácticas que buscan disminuir y controlar las eventualidades. Existe un cálculo más elaborado de los tiempos de viaje, de los horarios punta, de la hora de salida. Asimismo, quienes viajan en horarios de mayor tránsito han desarrollado estrategias y tienen un conocimiento de las rutas más expeditas o más seguras, conocen los atajos, desvíos y las pistas que deben tomar para evitar demoras. Se trata de un tiempo y espacio que se planifica, para el cual hay que prepararse. El saludo y la conversación también son prácticas relevantes que se ven amenazadas por la privatización del viaje. En este sentido, y de acuerdo a lo expresado por los entrevistados, se podría deducir que no saludarse o conversar habitualmente con otras personas durante el trayecto diario reduciría las instancias de sociabilidad en espacios públicos, promoviendo el aislamiento de la personas y podría derivar en un aumento de la sensación de desconfianza, inseguridad, e incertidumbre entre quienes viajan.

\section{CONSIDERACIONES FINALES: ¿MODERNIDAD VÍA SATURACIÓN?}

Un aviso publicitario que aparece en el principal diario talquino en 1976 anunciaba: "Hoy es el gran día. Honda, el automóvil que asombra al mundo por su avanzada tecnología, llega por fin a Talca” (La Mañana, 10 de febrero de 1976). El aviso denota aquella condición de periferia o de aislamiento respecto al proceso modernizador; da cuenta de la expectativa y la espera asociadas a la llegada de la ansiada modernidad y resalta su carácter exclusivo. 
Si bien históricamente en Chile la modernización ha representado un capital escaso que ha tendido a vincularse a territorios y grupos sociales acotados, las últimas décadas develan un cambio en el modelo. La misma ciudad de Talca que esperaba con ansias el primer automóvil Honda, ahora ve cómo sus calles se llenan de vehículos particulares, de colectivos y buses que ofrecen sus servicios de locomoción. Siguiendo una lógica de acción-reacción que tiene por modelo a la capital, las calles se pavimentan, se multiplican los caminos interurbanos, proliferan los semáforos, la señalética, los estacionamientos, los tags, las leyes y regulaciones.

Es el sistema de la automovilidad (Urry, 2004) en acción, con sus lógicas de dominación y su capacidad de auto-reproducción. El aumento acelerado del parque de vehículos motorizados durante los últimos ańos, no solo exige una transformación del entorno material y del paisaje de la ciudad -percibidos históricamente como modernos-, sino que promueve además nuevas sociabilidades en la experiencia del viaje cotidiano, pero también en la experiencia de la vida familiar y comunitaria, del trabajo, el ocio, etc. Así, aunque en principio este sistema de la automovilidad se incorpora a las prácticas tradicionales del lugar que guardan poca relación con los discursos modernizantes bajo los cuales se instalan, esto no significa que con el tiempo no existan consecuencias sobre estas prácticas. Aún cuando la pausa y las interrupciones todavía gobiernan el tiempo de los desplazamientos en Talca, la progresiva motorización de los viajes ha ido transformando el ritmo de las calles y la forma en que se percibe la ciudad.

Ante esta inminente mutación, cabría preguntarse por el rol que cabe al Estado. Ilustrativo en este sentido fue un seminario que reunió en Talca a diversas autoridades locales y académicos dedicados al estudio de la movilidad urbana durante noviembre de 2015. Las distancias parecían insalvables luego que el profesor Tim Edensor alabara las cualidades urbanas del barrio alrededor del terminal de buses de Talca, y atribuyera estas a la multiplicidad de usos simultáneos de la calle, a la informalidad, al comercio ambulante y al reducido control y regulación sobre los espacios.

Este entendimiento de la calle como espacio de confluencia de distintos medios, usos, e intereses pareciera ser completamente opuesto a la tendencia general que muestran las transformaciones en el entorno y las experiencias de viaje durante los últimos años en Talca, en donde prima la creciente regulación, la segregación vial, la apuesta por los dispositivos tecnológicos y los subsidios indirectos al viaje motorizado privado. En la vereda opuesta, la caminata como modo único de viaje, que predominaba entre quienes vivían en áreas aledañas al centro, ha perdido relevancia ayudado por el estado deplorable de veredas y la escasa iniciativa de las autoridades para invertir en mejoras peatonales más allá de las áreas centrales. El uso de la bicicleta, en una ciudad que históricamente ha destacado en Chile por el uso de este vehículo, muestra una preocupante tendencia a la baja. 
Alarmante, resulta por otra parte, la imposibilidad de frenar la rápida expansión horizontal de la ciudad en las últimas décadas y la reciente aprobación de un nuevo plano regulador que aumenta en tres veces el área urbana. Tampoco sorprende las numerosas inversiones que recientemente se ha efectuado en infraestructura vial, entre los que se encuentra la sincronización de la señalización luminosa, el ensanche y pavimentación de aceras o los intentos reiterados de expulsar el comercio ambulante de las calles del centro. Medidas como estas buscan implantar un modelo de movilidad urbana que prioriza por los flujos motorizados, con pretensiones de racionalidad y eficiencia, relegando a una situación subordinada otras formas de movilidad que tradicionalmente han primado.

La escasa reflexión o crítica sobre esta arremetida de los motores no es signo de una modernidad sin modernización (Bauman, 2008), ni tampoco de una modernidad como camino hacia la modernización (Gorelik, 2003), sino más bien el escenario refiere a una modernización sin reflexión, sin proyecto, ni un Estado que planifique y defina lineamientos las necesidades de localidad con los posibles ideales de ciudad. A esto se suma la mirada miope moderna desde los organismos técnicos que tradicionalmente han entendido la movilidad como un asunto racional, sin considerar sus implicancias psicológicas y sociales (Schiefelbusch, 2010).

Tal como lo anuncia Romero (2005) respecto al proceso de metropolización de las capitales latinoamericanas, resulta evidente que la variable del número (en este caso - de vehículos motorizados en circulación) tiene un lugar central en la transformación de la vida urbana. A mayor cantidad de vehículos motorizados, mayor es la presión sobre el territorio y el entorno construido, y mayor el impacto sobre las prácticas y la vida cotidiana. La incertidumbre se incrementa si se observan las posibles proyecciones de este mercado que hoy ubica a Chile con una tasa de 17,4 vehículos por 100 habitantes, mientras EE.UU. llega a 77,4 por 100 habitantes (ANAC, 2014).

Actualmente, quienes están preocupados de diseñar la ciudad disocian el espacio público del espacio del viaje. El espacio público se encuentra limitado a veredas, plazas y parques, pero no considera otros espacios móviles como son los paraderos y espacios intermodales y menos los vehículos sobre los cuales se desarrollan las rutinas diarias de gran parte de la población. Bicicletas, automóviles, colectivos o buses, etc., no solo son medios de movilización que permiten llegar de un lugar a otro, sino que son artefactos que median nuestra experiencia con el entorno recorrido. Cada uno implica formas distintas de contacto y permanencia con este entorno. En consecuencia, se vuelve urgente revisar cómo está relación ha cambiado en las últimas décadas. Si hace treinta años la experiencia común en una ciudad como Talca consistía en caminar de un lugar a otro y eso realzaba el rol de la arquitectura y del espacio de la calle como soporte directo de la experiencia 
cotidiana, actualmente es cada vez más habitual mediar esa experiencia a través vehículos u otras tecnologías.

Pensando desde la lógica de Ingold (2000), esta mediatización e instrumentalización de la experiencia del entorno, implicaría un distanciamiento entre las narrativas de viaje y los lugares que se recorren, lo que llevaría a la desafección y el desanclaje. ¿Es, por tanto, frente a un debilitamiento de la densidad de historias asociadas a estos lugares, privilegiándose su entendimiento como locaciones, espacios aislados o destinos? Este posible desanclaje o debilitamiento de la relación sensorial que el sujeto establece con los lugares en los que habita, implica, al fin y al cabo, el debilitamiento de los "lugares comunes" del sentimiento de colectividad.

\section{REFERENCIAS}

Asociación Nacional Automotriz de Chile A.G. [ANAC]. Anuario Automotriz 2014-2015, 2015.

Bauman, Zygmunt. Múltiples Culturas, una sola humanidad. Madrid: Katz Editores, 2008.

CEUT ."Encuesta de Caracterización Regional del Maule". Talca: Centro de Estudios Urbano Territoriales, Universidad Católica del Maule, 2014.

Comisión de Estudios Habitacionales y Urbanos (CEHU), Ciudades con calidad de vida. Diagnósticos estratégicos de ciudades chilenas: Informe de diagnóstico base: La Serena-Coquimbo. Santiago, Chile: Ministerio de Vivienda y Urbanismo, 2011.

Eltit, Verónica Xaviera. "Transporte urbano no motorizado: el potencial de la bicicleta en la ciudad de Temuco", Revista INVI, 72/26 (agosto de 2011): 153-184.

"Encuesta de Movilidad Cotidiana en Talca”. Realizada en el contexto del Fondecyt Regular 2013, No 1130695. Universidad Católica del Maule, 2014.

Errázuriz, Tomás. "La experiencia del tránsito. Motorización y vida cotidiana en el Santiago metropolitano, 1900-1931”, Tesis de Doctorado en Arquitectura y Estudios Urbanos, Pontificia Universidad Católica de Chile, 2010. 
García Canclini, Néstor. Imaginarios urbanos. Buenos Aires: Eudeba, 2010.

Giannini, Humberto. La "reflexión" cotidiana. Hacia una arqueología de la experiencia. Santiago, Chile: Editorial Universitaria, 2004.

Gorelik, Adrián. "Ciudad, modernidad, modernización”, Universitas Humanistica, (junio) número 056, Pontificia Universidad Javeriana, Bogotá, Colombia (2003): 11-27.

Greene, Ricardo (ed.). Ciudad Fritanga. Crónicas de ciudades no metropolitanas. Santiago, Chile: Editorial Bifurcaciones, 2014.

Ingold, Tim. The perception of the environment. Essays on livelihood dwelling and skill. London, New York: Routledge, 2000.

Instituto Nacional de Estadística [INE] "Compendios estadísticos de Transportes y Comunicaciones" (1975-2000); "Parque de Vehículos en Circulación" (2001-2012).

Jirón, Paola, Imilan, Walter y Iturra, Luis. "Relearning to travel in Santiago. The thickening of mobile place-making through travelling know-how", Cultural Geographies, Vol 23, Issue 4, 2016, pp. 599-614.

Joseph, Isaac. El Transeúnte y el espacio urbano. Barcelona: Gedisa, 2002.

Riveros, Sebastián. "Recopilación de antecedentes cuantitativos y espaciales que den cuenta de los cambios socio territoriales generados por la agroindustria en la relación urbano - rural en la Región del Maule”, Informe de práctica Profesional en el CEUT, 2013, Escuela de Geografía, FAU.

Romero, José Luis. Latinoamérica. Las ciudades y las ideas. Buenos Aires: Siglo XXI Editores, 2005.

Schiefelbusch, Martin."Rational planning for emotional mobility. The case of public transport development”, Planning Theory, 9 (2010): 200-222.

Simmel, Georg. "La metrópolis y la vida mental". Bifurcaciones [online]. núm. 4, primavera. World Wide Web document. Disponible en: www.bifurcaciones. cl/004/reserva.html [Consultado el 11 de septiembre de 2014]. ISSN 0718-1132, 2005. 
Superintendencia de Pensiones, "Ingreso imponible promedio por región y A.F.P. (al 30 de Junio de 2015)". Disponible en: http://www.safp.cl/safpstats/ stats/.si.php?id=inf_estadistica/aficot/trimestral/2015/06/32C.html [Consultado el 30 de septiembre de 2015].

United Nations, ONU-Habitat. Por un mejor futuro Urbano. United Nations Human Settlements Programme, 2014 [Folleto].

United Nations, World Urbanization Prospects. The 2014 Revision. Department of Economic and Social Affairs, New York, 2015.

Ureta, Sebastián. "Manejando por Santiago. Explorando el uso de automóviles por parte de habitantes de bajos ingresos desde una óptica de movilidad sustentable", Revista Eure XXXV/105 (agosto de 2009): 71-93.

Urry, John. "The system of automobility", Theory, Culture \& Society 21, 4/5 (2004): 25-39.

“El Pulso de la ciudad”, El Centro (Talca), 20 de abril de 2003, p. B3.

"Hoy es el gran día. Honda, el automóvil que asombra al mundo por su avanzada tecnología, llega por fin a Talca", La Mañana (Talca), 10 de febrero de 1976 , p. 8.

"Metamorfosis vial", El Centro (Talca), 02 de septiembre de 2004, p. 13. 